\title{
Ultrafast Technique of Impulsive Noise Removal with Application to Microarray Image Denoising
}

\author{
Bogdan Smolka ${ }^{1, \star}$ and Konstantinos N. Plataniotis ${ }^{2}$ \\ ${ }^{1}$ Silesian University of Technology, Department of Automatic Control, \\ Akademicka 16 Str, 44-100 Gliwice, Poland \\ bsmolka@ia.polsl.gliwice.pl \\ 2 The Edward S. Rogers Sr. Department of Electrical and Computer Engineering, \\ University of Toronto, 10 King's College Road, Toronto ON, M5S 3G4, Canada
}

\begin{abstract}
In this paper a novel approach to the impulsive noise removal in color images is presented. The proposed technique employs the switching scheme based on the impulse detection mechanism using the so called peer group concept. Compared to the vector median filter, the proposed technique consistently yields better results in suppressing both the random-valued and fixed-valued impulsive noise. The main advantage of the proposed noise detection framework is its enormous computational speed, which enables efficient filtering of large images in real-time applications. The proposed filtering scheme has been successfully applied to the denoising of the cDNA microarray images. Experimental results proved that the new filter is capable of removing efficiently the impulses present in multichannel images, while preserving their textural features.
\end{abstract}

\section{Introduction}

In this paper a novel approach to the detection and removal of impulsive noise in color images is presented. The main advantage of the described technique is its simplicity and enormous computational speed. The proposed method is using the well known vector median filter for the suppression of the detected noise, however different techniques can be used for the denoising of the previously detected impulses.

The cDNA microarray is a popular and effective method for simultaneous assaying the expression of large numbers of genes and is perfectly suited for the comparison of gene ex-

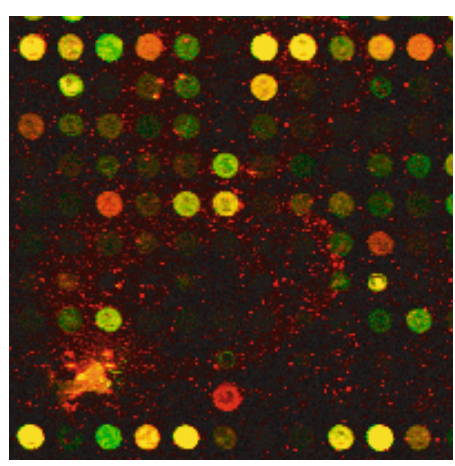

Fig. 1. Two-channel image of the cDNA microarray pression in different populations of cells. A microarray is a collection of spots containing DNA, deposited on the surface of a glass slide. Each of the spots contains multiple copies of a single DNA sequence.

\footnotetext{
* This research has been supported by the KBN grant 4T11F01824.
} 
The probes are tagged with fluorescent reporter molecules, which emit detectable light when stimulated by laser. The emitted light is captured by a detector, which records the light intensity. When the laser scans the entire slide, a large array image containing thousands of spots is produced. The fluorescent intensities for each of the two dyes are measured separately, producing a twochannel image, (Figs. 11, 8).

The intensities provided by the array image can be quantified by measuring the average or integrated intensities of the spots. However, the evaluation of microarray images is a difficult task as the natural fluorescence of the glass slide and non-specifically bounded DNA or dye molecules add a substantial noise floor to the microarray image. To make the task even more challenging, the microarrays are also afflicted with discrete image artifacts, such as highly fluorescent dust particles, unattached dye, salt deposits from evaporated solvents, fibers and various airborne debris. So, the task of microarray image enhancement and especially the removal of artifacts is of paramount importance, [1].

\section{Impulsive Noise Removal}

The majority of the nonlinear, multichannel filters are based on the ordering of vectors in a sliding filter window. The output of these filters is defined as the lowest ranked vector according to a specific vector ordering technique.

Let the color images be represented in the RGB color space and let $\mathbf{x}_{1}, \mathbf{x}_{2}$, $\ldots, \mathbf{x}_{n}$ be $n$ samples from the sliding filter window $W$. Each of the $\mathbf{x}_{i}$ is an $\mu$-dimensional multichannel vector, (in our case $\mu=3$ ). The goal of the vector ordering is to arrange the set of $n$ vectors $\left\{\mathbf{x}_{1}, \mathbf{x}_{2}, \ldots, \mathbf{x}_{n}\right\}$ belonging to $W$ using some sorting criterion.

The ordering based on the cumulative distance function $R\left(\mathbf{x}_{i}\right)$ is usually defined as: $R\left(\mathbf{x}_{i}\right)=\sum_{j=1}^{n} \rho\left(\mathbf{x}_{i}, \mathbf{x}_{j}\right)$, where $\rho\left(\mathbf{x}_{i}, \mathbf{x}_{j}\right)$ is a function of the distance among $\mathbf{x}_{i}$ and $\mathbf{x}_{j}$. The ordering of the scalar quantities according to $R\left(\mathbf{x}_{i}\right)$ generates the ordered set of vectors, [2].

One of the most important noise reduction filters is the vector median. In the case of gray scale images, given a set $W$ containing $n$ samples, the median of the set is defined as $x_{(1)} \in W$ such that $\sum_{j}\left|x_{(1)}-x_{j}\right| \leq \sum_{j}\left|x_{i}-x_{j}\right|, \forall x_{i}, x_{j} \in W$. Median filters exhibit good noise reduction capabilities, and outperform simple nonadaptive linear filters in preserving signal discontinuities.

As in many applications the signal is multidimensional, in [4] the Vector $M e$ dian Filter (VMF) was introduced, by generalizing the scalar median definition using a suitable vector norm denoted as $\|\cdot\|$. Given a set $W$ of $n$ vectors, the vector median of the set is defined as $\mathbf{x}_{(1)} \in W$ satisfying

$$
\sum_{j}\left\|\mathbf{x}_{(1)}-\mathbf{x}_{j}\right\| \leq \sum_{j}\left\|\mathbf{x}_{i}-\mathbf{x}_{j}\right\|, \quad \forall \mathbf{x}_{i}, \mathbf{x}_{j} \in W
$$

\section{Proposed Noise Detection Algorithm}

The main objective of the noise reduction algorithms is to suppress noise, while preserving important image features like edges, corners or texture. 
Over the years various impulsive noise reduction algorithms have been proposed, [5677. The main drawback of many standard filters is the fact that they fail to distinguish between the original uncorrupted pixels and pixels affected by the noise process, which leads to poor visual quality of the restored images.

This is also a serious drawback of the very popular Vector Median Filter. It is quite easy to notice that the VMF offers good performance in the removal of impulsive noise, but at the same time it introduces unnecessary changes to the pixels not corrupted by the noise process, which leads to extensive image blurring, destruction of image texture and even artifacts like artificial streaks. This behavior of the VMF can be easily observed in Fig. $2 \mathrm{~d}$ in which the the black pixels indicate those image pixels that were changed by the VMF algorithm. The test image was distorted by $5 \%$ random valued impulsive noise and the VMF replaced $80.7 \%$ of the image pixels.

Let us now modify the concept of the peer group introduced in [8] and extensively used in various filtering designs, mostly under the name of extended spatial neighborhood, 9 .

The peer group $\mathcal{P}\left(\mathbf{x}_{i}, m, d\right)$, in this paper will denote the set of $m$ neighbors $\mathbf{x}_{j}$ of the central pixel of $W$, which satisfy he following condition: $\left\|\mathbf{x}_{i}-\mathbf{x}_{j}\right\| \leq d$, $\mathbf{x}_{j} \in W, \mathbf{x}_{j} \neq \mathbf{x}_{i}$. In other words, the peer group $\mathcal{P}$ associated with the central pixel $\mathbf{x}_{i}$ of $W$ is a set of $m$ pixels which are in neighborhood relation with $\mathbf{x}_{i}$, whose distance to the central pixel is not exceeding $d$, (Fig. 3).

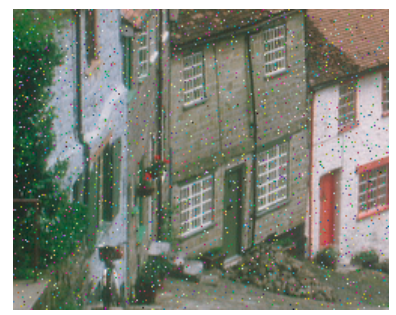

(a)

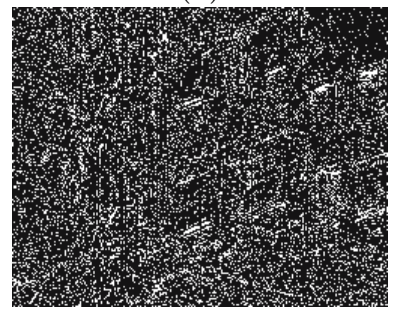

(d)

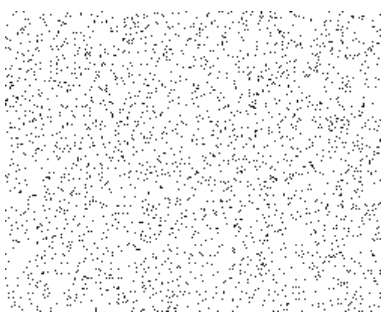

(b)

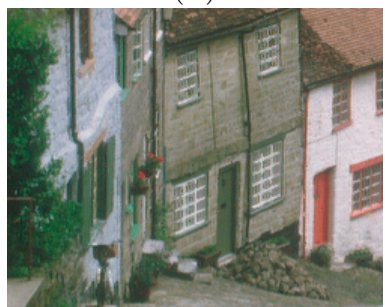

$(\mathbf{e})$

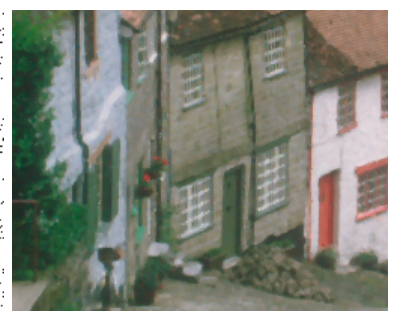

(c)

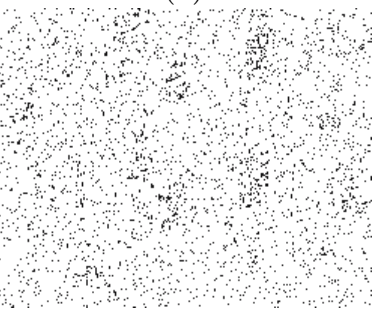

(f)

Fig. 2. Illustration of the efficiency of the new filtering design: a) test image GOLDHILL contaminated by random valued impulsive noise of $p=5 \%$, b) black dots show the pixels disturbed by noise, c) image restored with VMF, d) difference between the original and the VMF output, e) new filter output, $(m=3, d=50)$ and besides the corresponding residual image $\mathrm{f}$ ) 
The proposed impulsive noise detection algorithm works as follows: if there exists a peer group $\mathcal{P}\left(\mathbf{x}_{i}, m, d\right)$, which means that at least $m$ neighbors of $\mathbf{x}_{i}$ satisfy the closeness condition, then the pixel $\mathbf{x}_{i}$ is treated as not corrupted by noise, otherwise it is declared to be noisy and can be filtered with any efficient noise reduction algorithm.

As the output is switched between the identity and a filtering operation, various filtering designs can be used instead of the VMF, [5]. In this paper we have chosen the VMF mainly to demonstrate the efficiency and extremely low computational effort of the proposed noise detection framework.

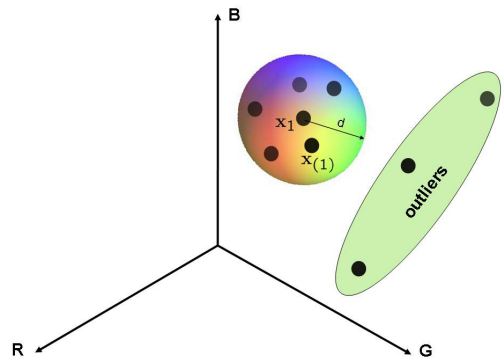

Fig. 3. The concept of the peer group centered at $\mathbf{x}_{1},(m=5)$

The low computational complexity stems from the fact that when the peer group parameter $m$ is low, for example $m=2$, then if the algorithm finds two pixels, which are close enough to the central pixel under consideration, the pixel $\mathbf{x}_{i}$ is declared as noisefree and the sliding window moves to the adjacent pixel. Very often only a few calculations of the distances $\left\|\mathbf{x}_{i}-\mathbf{x}_{j}\right\|, \mathbf{x}_{j} \in W$ are needed to classify the pixel as undisturbed by noise. The minimal number of calculation of the distances needed to classify the pixel is thus equal to $m$ and the maximal number of distances is $n-m$, where $n$ is the number of pixels contained in the filtering window $W$. The number of distances needed for the detection of impulses is extremely low when compared with the number of distances needed by the VMF algorithm which is equal to $n(n-1) / 2$.

\section{Simulation Results}

In many practical situations, images are corrupted by noise caused either by faulty image sensors or due to transmission errors resulting from man-made phenomena such as ignition transients in the vicinity of the receivers or even natural phenomena such as lightning in the atmosphere.

The impulsive noise is often generated by bit errors, especially during the scanning or transmission over noisy information channels. In this paper the noisy signal is modelled as $\mathbf{x}_{i}=\left\{x_{i 1}, x_{i 2}, x_{i 3}\right\}$, where $x_{i k}=v_{i k}$ with probability $\pi$ and $o_{i k}$ (original, undisturbed value) with probability $1-\pi$, and the contamination component $v_{i k}$ is a random variable. We will assume two models, which will be called impulsive salt \& pepper or fixed-valued noise, when $v_{i k}=\{0,255\}$ and impulsive uniform or random-valued noise, when $v_{i k} \in[0,255]$. It can be noticed that the first model is a special case of the uniform noise, as this noise can take on only two values 0 or 255 with the same probability, assuming 8-Bit per channel, color image representation.

In both noise models the contamination of the color image components is uncorrelated, and the overall contamination rate is $p=1-(1-\pi)^{3}$. For the measurement of the restoration quality the commonly used Root Mean Square 
(a)

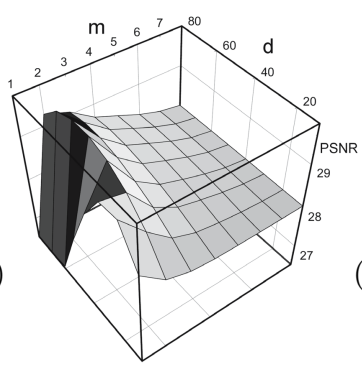

(d)

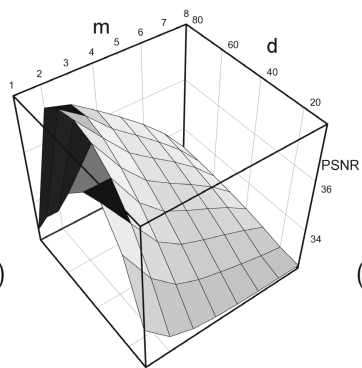

(b)

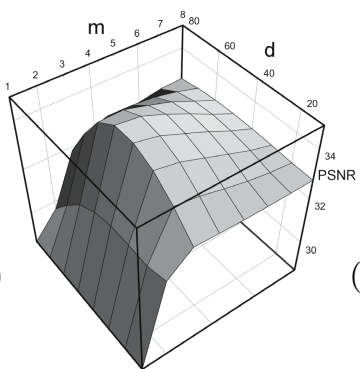

(e)

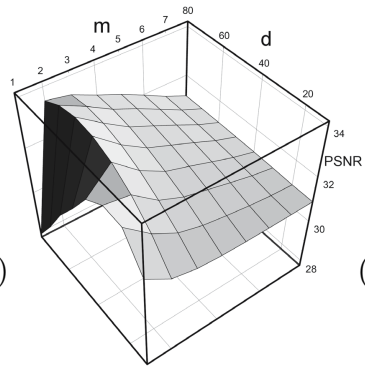

(c)

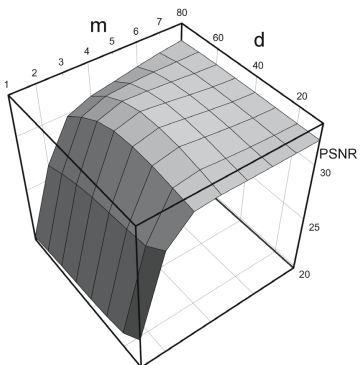

(f)

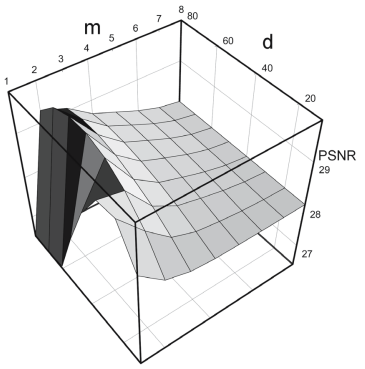

Fig. 4. Dependence of the PSNR on parameters $m, d$ for the LENA image contaminated by salt $\&$ pepper (a-c) and uniform (d-f) impulsive noise for $p=10,20,30 \%$

Error (RMSE) expressed through the Peak Signal to Noise Ratio (PSNR) was used, as the RMSE is a good measure of the efficiency of impulsive noise suppression. The PSNR is defined as

$$
P S N R=20 \log _{10}\left(\frac{255}{\sqrt{M S E}}\right), M S E=\frac{\sum_{i=1}^{N} \sum_{k=1}^{\mu}\left(x_{i k}-o_{i k}\right)^{2}}{N \mu},
$$

where $N$ is the total number of image pixels, and $x_{i k}, o_{i k}$ denote the $k$-th component of the noisy image pixel channel and its original, undisturbed value at a pixel position $i$, respectively.

The parameters $m$ and $d$ provide control over the performance of the impulsive noise detection process. For its assessment a series of simulations on natural images was performed.

With regard to the parameter $m$ of the peer group $\mathcal{P}$ the simulation results show that when the contamination intensity is low, good results are achieved for $m=2$ in case of both the fixed valued and impulsive noise, (Figs. 4 a,d). For higher noise probability $p$, the images contaminated by fixed valued impulsive noise require $m=3$, (Figs. 4 b,c). Surprisingly, good results are achieved for $m=2$, when the images are contaminated by random valued noise, (Figs. 4 4 e,f). As the filtering results are not very sensitive to the choice of $m$, we used $m=3$ for the comparisons with the VMF.

The experiments conducted on a broad variety of natural color images have shown, 10[11 that the parameter $d$ should be equal to about 50, (Fig. 6) as such 
a setting guarantees good performance of the proposed switching scheme, independently on the image characteristics, noise model and contamination intensity.

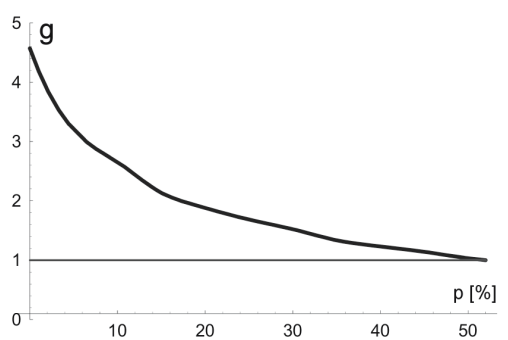

Fig. 5. Filtering efficiency of the proposed noise removal algorithm in comparison with the VMF for salt $\mathcal{E}$ pepper noise for LENA image with $d=50$ and $m=3$

shows that the new filter rejects the impulses and replaces only a small fraction of the undisturbed pixels, (in this example the contamination intensity was $p=5 \%$ and only $6.7 \%$ of the pixels were replaced by the VMF).

(a)

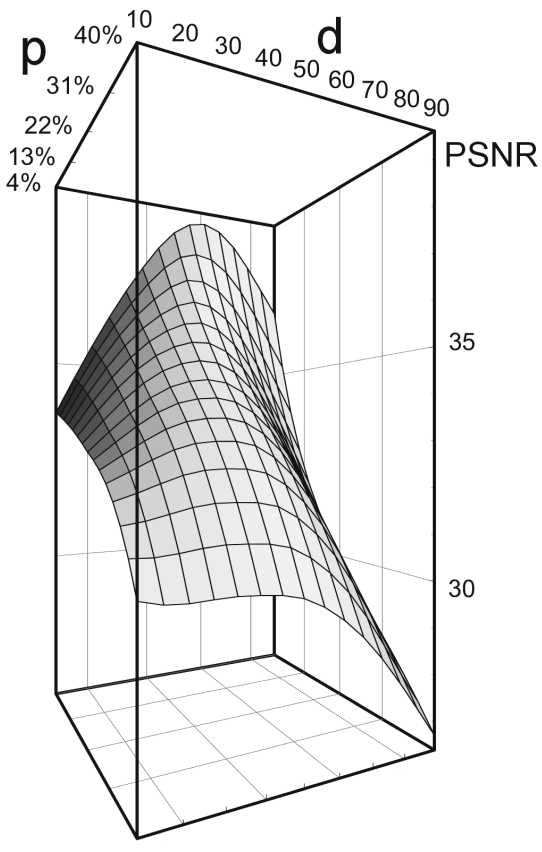

The main advantage of the proposed noise detection technique is its enormous computational speed. The comparison with the VMF, presented in Fig. 5 shows that the new technique is for low contamination intensities 2-4 times faster than the VMF.

The efficiency of the proposed technique can be observed in Fig. 77, in which zoomed parts of the test color images were distorted by uniform impulsive noise and restored with VMF and with the new filter. As can be observed the incorporated switching scheme enables the preservation of edges and fine image details. This behavior is also confirmed in Fig. 2 f,g, which 

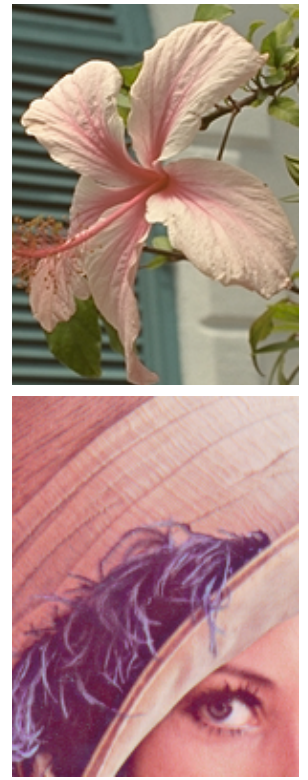

(a)
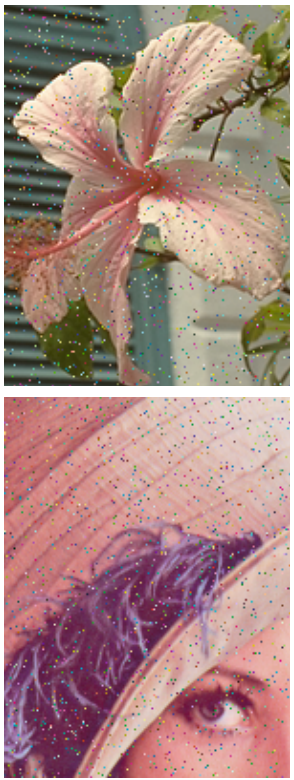

(b)
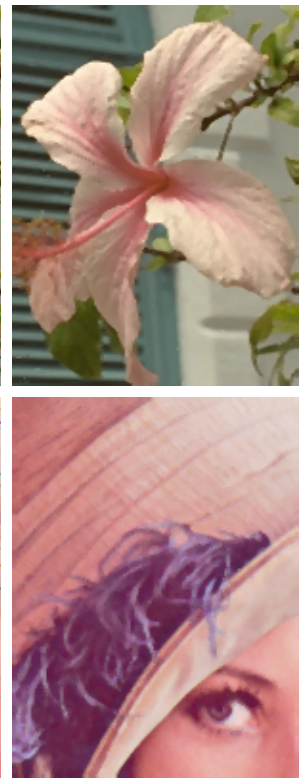

(c)
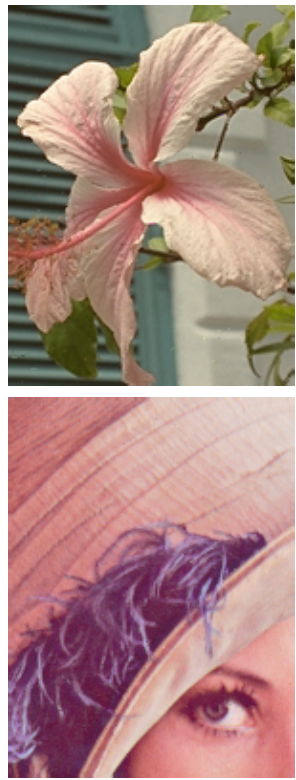

(d)

Fig. 7. Illustrative examples of the filtering efficiency: a) zoomed parts of the color test images, b) images contaminated by $5 \%$ uniform noise, c) restoration achieved with the $\mathrm{VMF}, \mathbf{d}$ ) filtering results achieved using the new noise detection technique

(a)

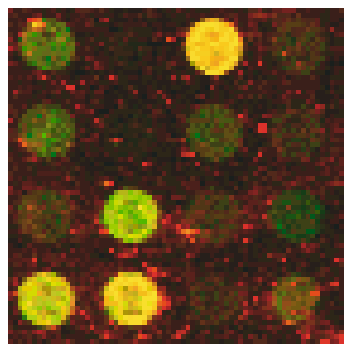

(b)

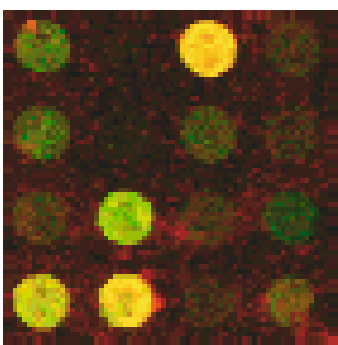

(c)
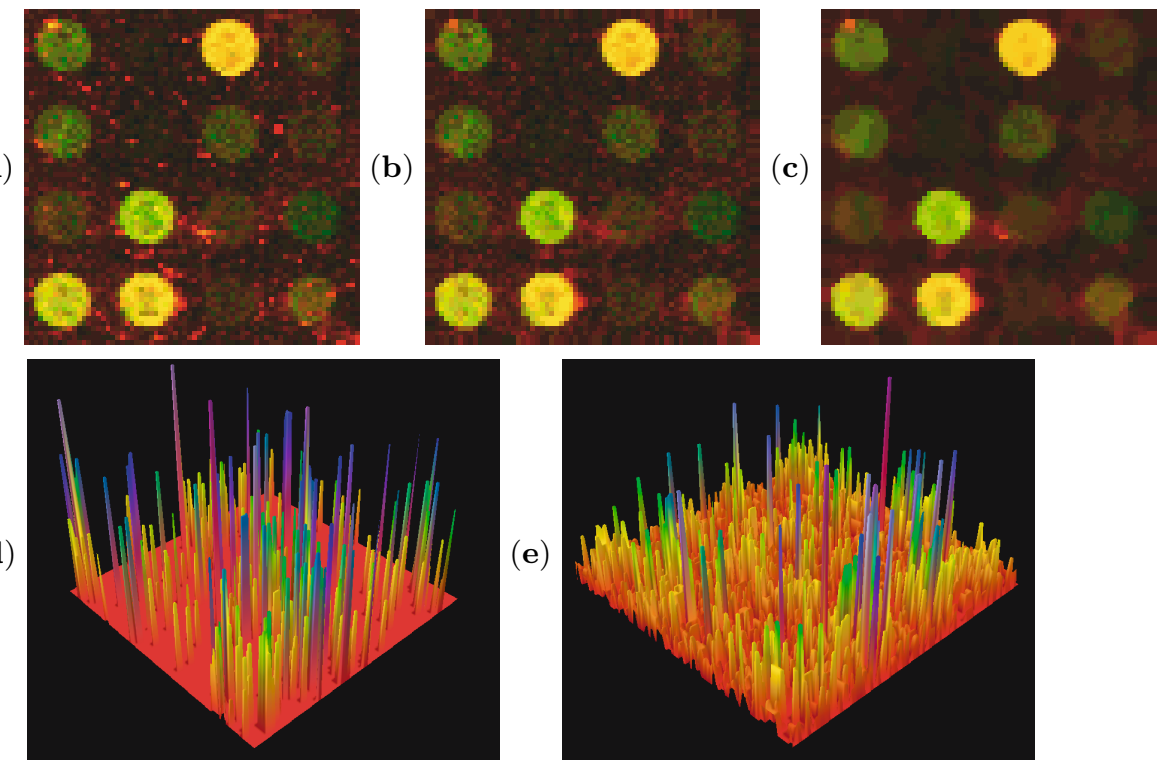

(e)

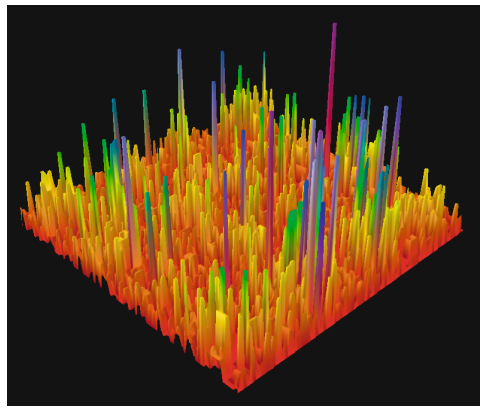

Fig. 8. Filtering efficiency on the cDNA images: a) noisy image, b) restored with the new technique, $\mathbf{c}$ ) restored with $\mathrm{VMF}, \mathbf{d}$ ) visualization of the difference between the noisy image and its restored version corresponding to the new filter, e) error images corresponding to VMF, (see also Fig. 2) 
The good performance of the proposed switching scheme can be also observed in Fig. 8, which depicts the results of impulsive noise suppression in cDNA microarrays. It can be noticed that the proposed filter removes the spikes only, while preserving the textural information needed for the assessment of the spots intensity. This behavior enables better evaluation of the spots intensities as the impulsive noise is efficiently removed and does not affect the measurements of the mean spot's intensity. Additionally the removal of impulses and small clusters enables further filtering of the Gaussian noise with fast linear techniques.

\section{Conclusion}

In this paper a new approach to the problem of impulsive noise detection and removal in color images has been presented. The main advantage of the proposed technique is its extraordinary high computational speed, which makes it attractive for real-time applications and denoising of very large images, like the cDNA microarrays. The noise detection scheme has been coupled in this paper with the vector median filter, however the computational speed can be further increased when employing a less computationally demanding noise removal algorithm.

\section{References}

1. Lukac, R., Plataniotis, K.N., Smolka, B., Venetsanopoulos, A.N.: A Multichannel Order-Statistic Technique for cDNA Microarray Image Processing. IEEE Transactions on Nanobioscience, Vol. 3, No. 4, (2004) 272-285

2. Lukac, R., Smolka, B., Martin, K., Plataniotis, K.N., Venetsanopoulos, A.N.: Vector Filtering for Color Imaging. IEEE Signal Processing Magazine, Special Issue on Color Image Processing, Vol. 22, No. 1, (2005) 74-86

3. Lukac, R.: Adaptive Vector Median Filtering. Pattern Recognition Letters. Vol. 24, No. 12, (2003) 1889-1899

4. Astola, J., Haavisto, P., Neuvo, Y.: Vector Median Filters. Proceedings of the IEEE, Vol. 78, (1990) 678-689

5. Plataniotis, K.N., Venetsanopoulos, A.N.: Color Image Processing and Applications. Springer Verlag, (2000)

6. Lukac, R.: Color Image Filtering by Vector Directional Order-Statistics. Pattern Recognition and Image Analysis, Vol. 12, No. 3, (2002) 279-285

7. Lukac, R.: Vector LUM Smoothers as Impulse Detector for Color Images. Proceedings of European Conference on Circuit Theory and Design (ECCTD'01) in Espoo, Finland, August 28-31, III, (2001) 137-140.

8. Kenney, C., Deng, Y., Manjunath, B.S., Hewer, G.: Peer Group Image Enhancement. IEEE Trans. Image Processing, Vol. 10, No. 2, (2001) 326-334

9. Kober, V., Mozerov, M., Alvarez-Borrego, J: Nonlinear Filters with SpatiallyConnected Neighborhoods. Optical Engineering, Vol. 40, No. 6, (2001) 971-983,

10. Smolka, B., Plataniotis, K.N., Chydzinski, A., Szczepanski, M., Venetsanopulos, A.N., Wojciechowski, K.: Self-Adaptive Algorithm of Impulsive Noise Reduction in Color Images. Pattern Recognition, Vol. 35, (2002) 1771-1784

11. Smolka, B., Lukac, R., Chydzinski, A., Plataniotis, K.N., Wojciechowski, K.: Fast Adapive Similarity Based Impulsive Noise Reduction Filter. Real-Time Imaging, Vol. 9, No. 4, (2003) 261-276 